\title{
Microfluidic environment and tracking analysis for the observation of Artemia Franciscana
}

\author{
Jose Alonso Solis-Lemus ${ }^{1}$ \\ Jose.Solis-Lemus@city.ac.uk \\ Yushi Huang ${ }^{2}$, \\ s3440897@student.rmit.edu.au \\ Donald Wlodkowic ${ }^{2}$ \\ donald.wlodkowic@rmit.edu.au \\ Constantino Carlos Reyes-Aldasoro ${ }^{1}$ \\ reyes@city.ac.uk \\ http://staff.city.ac.uk/ sbbk034/
}

\author{
${ }^{1}$ Biomedical Engineering Research \\ Group, School of Mathematics, \\ Computer Science and \\ Engineering, City University \\ London, London EC1V OHB, UK
}

\begin{abstract}
The movement of small animals in well-defined environments is increasingly used in ecotoxicology as a sensitive indicator of water contamination with toxicants. This work describes a framework for the analysis of Artermia franciscana within miniaturised chambers. This work combines: (a) a proof-ofconcept miniaturised Lab-on-a-Chip (LOC) platform where any changes in swimming patterns exhibited by Artemia franciscana can be related to the toxicity of the environment with (b) a pipeline of image processing steps for the movement analysis of video sequences of test specimens. The analysis pipeline is semiautomatic as it allows a user to define a region of interest and confirm intensity levels that are used for two-level hysteresis segmentation. Tracking is automatic, and a large number of statistical measurements and visualisation tools are provided. In addition, proofreading tools for validation of tracks are provided to link, break or delete tracks. The software consists of Matlab ${ }^{\circledR} \mathrm{m}$-files and is available in the website http://www.objecttrack.org.uk/.
\end{abstract}

\section{Introduction}

There is an increasing trend to look for more ethically acceptable and also much more sensitive tests in ecotoxicology [1]. The latter are required to provide alternatives to the "conventional" mortality tests performed using selected aquatic test species such as marine crustaceans Artemia sp. This work demonstrates development of a proof-ofconcept technology for rapid assessment of sub-lethal acute toxic effects by means of impact of the toxicant on swimming behaviour of test organisms [2]-[4]. In contrast to conventionally performed end-point analysis based on counting numbers of dead/immobile specimens we performed time-resolved experiments using short clips of movies acquired at a high frame rate rather than static time-lapse photos. 


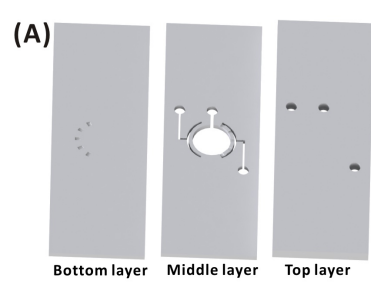

(D)

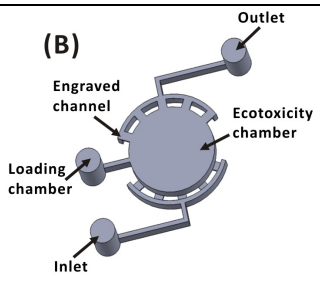

(E)

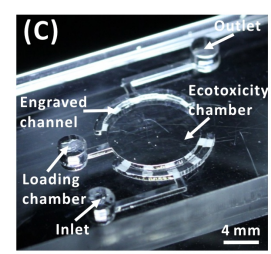

(F)
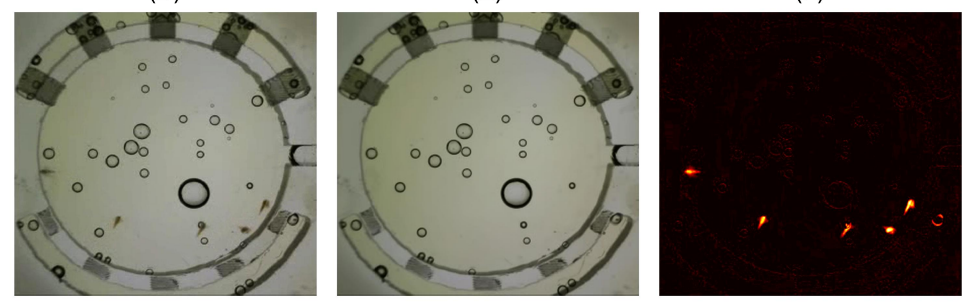

Figure 1: (a-c) Details of the fabrication of the Ecotoxicity chamber, for description see text. (d) One representative image of Artemia franciscana contained within a microfluidic system. The image is part of a sequence of 1,800 time frames and contains 5 animals and artefacts (bubbles of different sizes). Notice that one of the Artemia is crossing over one artefact. (e) The mean image of the video sequence. (f) The resulting image after the preprocessing steps. Notice how the five animals have been highlighted from the background, and also an artefact caused by a moving bubble at the right hand side.

Subsequently, we have performed video data analysis based on a custom software framework that enables us to visualise movements, and analyse quantitatively the experiments. Using this approach we are able to demonstrate rapid changes of behaviour of test specimens by analysing changes in speed of movement or distance travelled by each animal. This approach enabled us to increase sensitivity of the ecotoxicological analysis by including very sensitive sub-lethal behavioural endpoints.

\section{Materials and Methods}

\subsection{Materials}

Cysts of a marine crustacean Artemia franciscana (Ocean Nutrition Asia Co., Ltd., Thailand) were hatched at $24^{\circ} \mathrm{C}$ in a petri dish filled with filtered sea water with exposure to a light source (1000-4000 lux) for 30 hours before use.

A microfluidic system consisted of an innovative 3D multilayer chip-based device fabricated in a biologically compatible and optically transparent PMMA polymer using infrared laser micromachining [5]. The chip contained a specially designed micro-culture chamber with dimensions $(10 \mathrm{~mm}$ in diameter and $1.5 \mathrm{~mm}$ in height) optimised to hold multiple specimens of freely swimming crustacean larvae for up to 24 hours under continuous perfusion. Larvae of approximately 30 hours post hatching (hph) were loaded on chip-based devices. Microfluidic devices were perfused at a flow rate of $5 \mathrm{~mL} / \mathrm{h}$ with varying doses of potassium dichromate $(0 \mathrm{mg} / \mathrm{L}$ to $900 \mathrm{mg} / \mathrm{L}$; Sigma-Aldrich, Australia). The 3D multilayer chip contained a specially designed circular ecotoxicity chamber of 10 
$\mathrm{mm}$ in diameter and $1.5 \mathrm{~mm}$ in height, optimized to hold multiple specimens of freely swimming crustacean larvae for up to 24 hours under continuous microperfusion (Fig. 1a). Inlet and outlet channels were connected to the chamber through ten laser-ablated microchannels with a height of proximately $0.2 \mathrm{~mm}$ and positioned at the circumference of the chamber (Fig. 1b). The dimensions of ablated inlet and outlet ports enabled effective "caging" of free swimming inside the chamber. In order to improve mass transfer, microchannels were ablated in opposite different layers to provide a fluid flow across vertical plane of the microchamber (Fig. 1c). This design also reduced the blockage of the perfusion system by immobilized or dead organisms. The design incorporated an auxiliary manifold directly connected to the "caging" chamber. It enabled rapid and efficient loading of test specimens.

\subsection{Quantitative analysis}

Dose-dependent immobilisation of Artemia larvae in response to potassium dichromate was scored using an semi-automatic segmentation, tracking and analysis based on the software objectTrack (http://www.objectTrack.org.uk). This software is derived from a similar software used to analyse fluorescent neutrophils called PhagoSight [6]. The results were validated by two additional methods. First, manual counting of immobile larvae at 24-hour end-point was performed according to a standard Artoxkit-M biotest (MicroBioTests Inc, Belgium) standard operating protocol. Second, a new test protocol was developed to dynamically detect sub-lethal behavioural end-points. Accordingly, 15 seconds long videos were captured every hour for up to 24 hours of biotest duration using an embedded and miniaturised USB-based digital microimaging system (AM7013MT Dino-Lite Premier, DinoLite Ltd, Taiwan). The microscope was equipped with a 5.0-megapixel colour CMOS sensor (SXGA), variable magnification of up to 200x and numerical aperture (NA) of 0.135 .

The system had a capability to automatically acquire up to $30 \mathrm{fps}$ during videomicroscopy recording. The lighting was provided by 8 integrated ultra-bright white LEDs controlled by the dedicated software (DinoCapture2.0, Dino-Lite). The USB microscope was powered and controlled through a USB2.0 interface with an external PC computer running Windows 7 operating system (Microsoft, USA). The software interface supported fully programmable time-resolved data acquisition including direct control of both video capture and LED illumination.

\subsection{Movement analysis}

The image sequences contained the miniaturised chambers, the Artemia themselves, as well as numerous artefacts, mainly bubbles that can be seen in Fig. 1d. Removal of these artefacts was done in two stages. First, a mean image along all the time points of the video was calculated. In this way, those pixels crossed by moving objects tended to the mean value and the moving Artemia were removed (Fig. 1e). Subsequently, this mean image was removed from each image; negative values were rectified to provide an image where the regions different from the mean were highlighted. The subtraction of the mean removes the shading, which is common in bright field microscopy [7]. Two potential complications arose from this step: (a) when an Artemia is moving over an artefact, the artefact will be deleted from the body of the Artemia, (b) some air bubbles were drifting along time and therefore appeared as moving objects. The overlap described in (a) was 
corrected by applying a closing morphological operator [8] with a circular kernel and a low pass

(a)

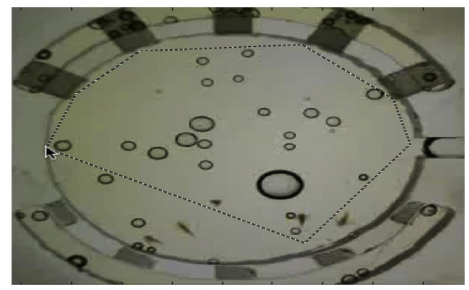

(b)

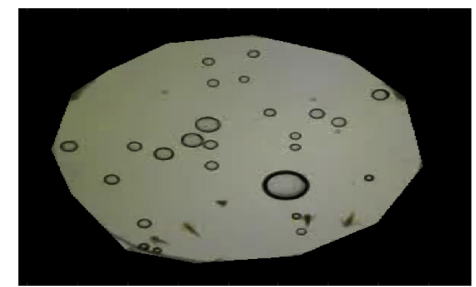

Figure 2: (a) Selection of points for the user-defined region of interest, not all points have been selected yet. (b) Final mask. Only the region inside will be considered for the analysis.

filtering with a Gaussian filter. In this way, the Artemia were not artifactually divided into two objects. The correction can be observed in the central Artemia in Fig. 1. The artefacts caused by bubbles in (b) (visible in the right hand side of Fig. 1f) can be removed in two ways: a mask to define the inner part of the chamber can be pre-defined, or the track generated by this object can be removed with the proofreading tools of the software. For this paper we opted for deploying a user-defined region of interest (Fig. 2) in which the user selects the mask, and all pixels outside the region are ignored. The result of the pre-processing steps is a series of images that resemble fluorescentlylabelled objects as the background will be dark and the objects will be bright and thus can be segmented by intensity.

The segmentation, tracking and analysis of fluorescently labelled objects have been described previously for neutrophils in zebrafish [6]. In this work we have adapted the algorithms described in [6], but briefly, a hysteresis threshold inspired by the Schmitt Trigger [9] was used to segment the Artemia larvae into objects. A low threshold discarded the background and a high threshold identified those regions that corresponded to foreground (i.e. the Artemia) with a higher certainty. The regions in between thresholds were assigned to one class or the other, depending on the proximity to the previously defined regions; if in contact with foreground, these became foreground, otherwise they were assigned to background. The thresholds were initially calculated with Otsu's algorithm [10] and verified by the user. Collisions between objects were analysed by volume, when an object increased considerably the volume (mean $+3 \times$ std) these were split into two by successive erosion. Tracking was performed with a keyhole model of movement [11] in which two regions of probability are defined for the landing of a moving object, a circle (for random movements) and a wedge (for directed movements), which together resemble a keyhole. Tracks that were separated by one or two time frames, but within a small distance were assumed to correspond to the same moving object, where the object disappeared between time frames, due to low intensity, and were linked.

Post-processing verified the tracks and provided visualisation and proofreading tools that allowed the following correction tools: breaking, joining and deleting tracks. ObjectTrack provides numerous statistical measurements such as average distance and maximum distance per track. Statistical measure like these ones have previously been used to measure neutrophil recruitment and behaviour [12], [13]. The tracking algorithm was compared against ICY [14] in [6] with comparable results 
A measurement of particular interest for the analysis of Artemia is the number of complete 360-degree turns an individual can describe. This measurement can help distinguish, linear, circular or zigzag patterns of movement [15].

(a)

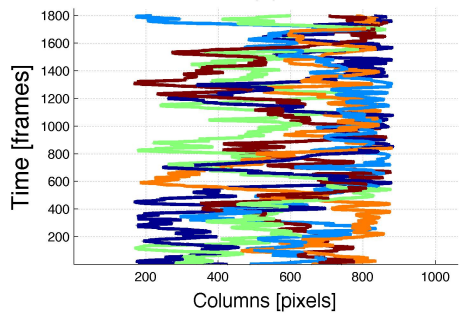

(c)

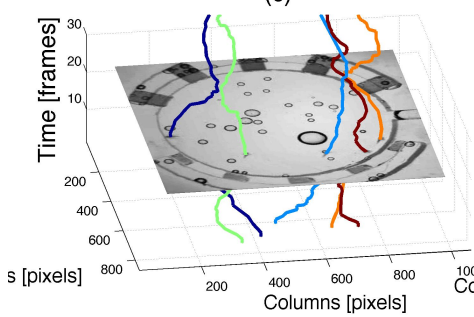

(b)

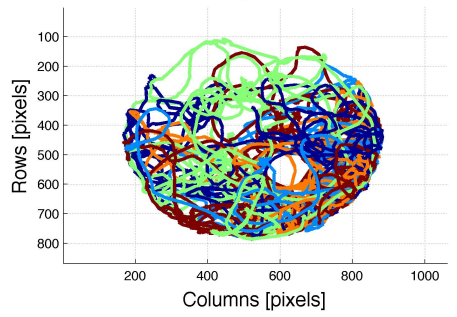

(d)

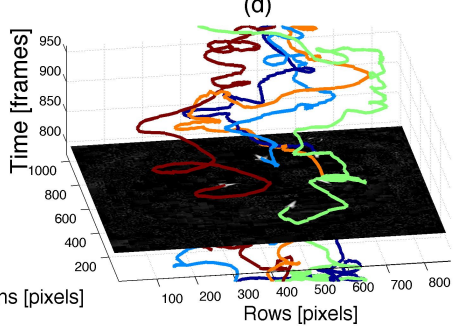

Figure 3: Visualisation of the tracks obtained from a video with 1,800 time frames. The tracks are color-coded for visualisation purposes; the visualisation tools can colour-code according to length or velocity. (a) Side view. Notice how the Artemia tend to swim more on the right hand side. (b) Top view. Notice how the Artemia concentrate on the bottom side of the environment and in the majority of the cases avoid the large bubble in the centre, except for one track that crosses it. (c) A zoom into the first 30 time frames; visualisation with tracks and images of the data can be easily obtained. (d) A zoom into the middle of the time frames with one slice of the pre-processed images overlaid. Notice how one of the tracks describes full 360-degree rotations.

This was calculated as following, for each "hop" between time frames the angle of

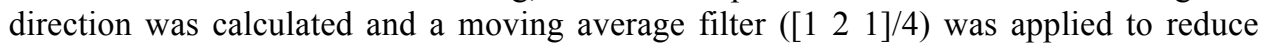
noise. The cumulative angle from the start was calculated and every time the cumulative angle crossed a threshold of $\pm 2 \pi$ a 360 -degree was counted, the next counting would need to cross again a threshold $\pm 2 \pi$ from the previous angle (Fig 4).

ObjectTrack is freely available as Matlab ${ }^{\circledR} \mathrm{m}$-files under the GNU General Public License. Synthetic data sets and a comprehensive user manual are available at the website http://www. objectTrack.org.uk.

\section{Results}

Videos of moving Artemia were analysed with the algorithm previously described. Fig. 3 shows the tracks corresponding to the video from which Fig. 1 was extracted. The lines describe the position of five Artemia individuals within 1,800 time frames. Fig. 3a shows the tracks with the time in the vertical dimension and columns of the image in the horizontal dimension. Fig. $3 b$ has been rotated so that the view angle is from the zenith and only rows and columns are visible. Each view can provide different information. For instance, Fig. 3a shows at which moment in time a track is in certain region. 
On the other hand, from Fig. $3 \mathrm{~b}$ it is clear that the region that corresponds to the larger bubble is rarely traversed by the Artemia, as there is only one crossing over it. Figs. 3c,d zoomed into some regions of the tracks and illustrates how a slice of the original or the pre-processed data can be overlaid. Notice the 360-degree turns that appear in Fig. 3d. Videos of the Artemia and the tracks can be easily generated with objectTrack.

Alternative visualisations are shown in Fig. 5a. Here, one track has been selected and the distance from the origin (Fig. 5a top) and the angle at each time frame (Fig. 5b centre) have been calculated.

The angle is then used to calculate when whole 360-degrees turns occur. These turns are highlighted with red stars overlaid on the plot and the total number counted. A comparison between tracks is shown in Fig. 5a bottom where the distance from origin of all tracks is displayed. As described in 2.2, immobile larvae were manually counted at the 24-hour end-points, while for software analysis total moving distances were used to evaluate the effect of the toxicant (Fig. 5b).

\section{Discussion}

In this work, we demonstrate development of a proof-of-concept technology for rapid assessment of sub-lethal and acute ecotoxic effects. An earlier version of this work was presented in [16]. The system working principles incorporated a time-resolved video data analysis to dynamically assess impact of the reference toxicant on swimming behaviour of marine crustacean Artemia franciscana.

We successfully adapted the segmentation and tracking algorithms of the package PhagoSight into objectTrack, which contains the pre-processing steps required to analyse light images, removed artefacts and generated fluorescent-like images to be then segmented and tracked.

There are several advantages of the combination of the technology and the computational framework. The algorithms allowed us to track rapid variations of swimming behaviour of Artemia larvae by analysing changes in their speed of movement, distance travelled or number of turns experienced by each animal. In addition it is possible to analyse more than one organism as compared with other settings where only one is observed [17]. This approach enabled us to both automate and significantly increase sensitivity of Artemia toxicity biotests as compared to a current gold standard that is based on manual counting of immobile larvae at 24-hour end-point.

The controlled microfluidic environment for the culture of Artemia larvae supports careful monitoring of the physic-chemical conditions, which is not possible in static conditions commonly employing multiwell plate-based culture.

With regards to the segmentation, it is important to notice that a cleaner image will result in a better segmentation, that is, even with the pre-processing steps, it may be possible for an animal to be lost if it crosses a large artefact with the same shades as the animal itself. The results shown in Fig. 5b show the same trend in a reduction of movement between the automated and manual procedures, albeit with a different rate of decrease, especially with concentrations above $56 \mathrm{mg} / \mathrm{L}$. It is assumed by the authors that the reason for the difference is the following. For manual counting, when an Artemia indicates a subtle movement, like the swing of an arm or the agitation of the body, it is regarded as a living animal. However, the tracking calculates the distance of movement between the centroids of the animal at consecutive time frames. Therefore, the animals do 
not appear to be displaced whilst they can still show movement. More interestingly, it can be seen that there is an increase in moving distance at a low concentration of $28 \mathrm{mg} / \mathrm{L}$.

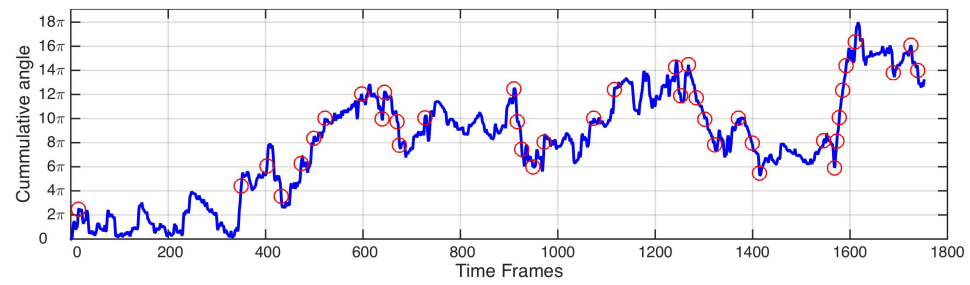

Figure 4: Illustration of the calculation of the 360-degree turns. The blue line indicates the cumulative angle displayed by a moving object and the red circles each time it crosses a threshold of 360 degrees.

(a)

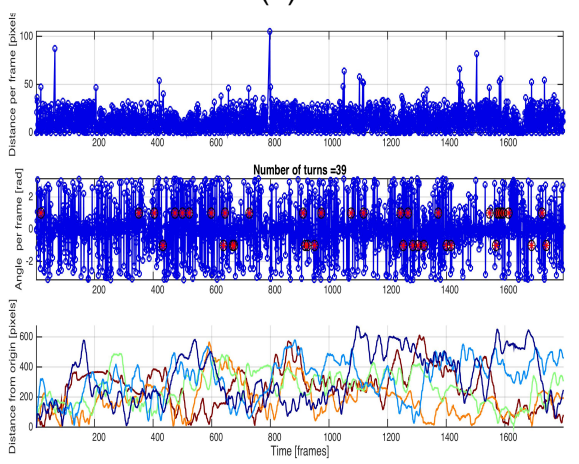

(b)

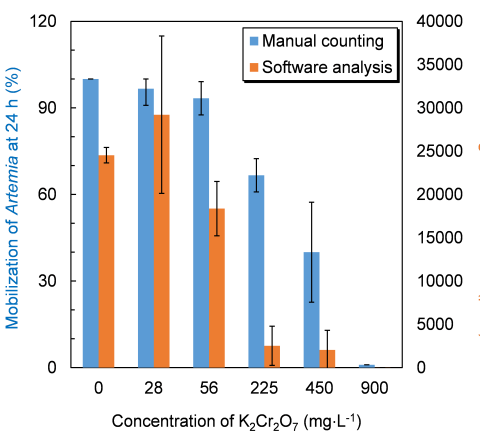

Figure 5: (a) Illustration of visualisation of measurements. (top) Distance covered by one Artemia at every hop between frames. This view is useful to detect higher or lower activity of one individual, (i.e. intoxication leading to death). (centre) Angle at every time frame. The positions at which a complete 360-degree turn occurs are marked (circle with a star); the relative vertical position corresponds to clockwise or counter clockwise turns. (bottom) Distance covered from the origin for all track. (b) Comparison between manual (blue columns, left) and automatic counting (orange columns, right). See text for explanation of the measurement differences.

It indicates that low concentration of potassium dichromate might have irritation effect on Artemia, which is worth of further study. Moreover, significant decrease of moving distance can be seen at concentration of $56 \mathrm{mg} / \mathrm{L}$ whilst the mobility rate is still close to $100 \%$. Similar results could also be found at $225 \mathrm{mg} / \mathrm{L}$ and $450 \mathrm{mg} / \mathrm{L}$, which Artemia had almost no movement with such concentration but still the mobility rate are higher that $60 \%$ and $30 \%$. Thus we believe that using moving distance to evaluate the toxicity effect could be more sensitive than simply using mobilization rate.

We intend to generate new measurements of change that take into account these conditions. In closing, we anticipate that video analysis algorithms combined with innovative chip-based microfluidic technologies will revolutionise ecotoxicological biotests by including very sensitive sub-lethal behavioural endpoints. We have numerous kinetic results not included in this work due to space limitations. 


\section{References}

[1] G. Persoone and P. G. Wells, 'Artemia in aquatic toxicology: a review', in Artemia Research and its applications, vol. 1: Morphology, Genetics, Strain Characterization, Toxicology, Wetteren, Belgium, 1987, pp. 259-275.

[2] K. I.-K. Wang, Z. Salcic, J. Yeh, J. Akagi, F. Zhu, C. J. Hall, K. E. Crosier, P. S. Crosier, and D. Wlodkowic, 'Toward embedded laboratory automation for smart lab-on-a-chip embryo arrays', Biosens. Bioelectron., vol. 48, pp. 188-196, Oct. 2013.

[3] D. Wlodkowic, K. Khoshmanesh, J. Akagi, D. E. Williams, and J. M. Cooper, 'Wormometry-on-a-chip: Innovative technologies for in situ analysis of small multicellular organisms', Cytom. Part J. Int. Soc. Anal. Cytol., vol. 79, no. 10, pp. 799-813, Oct. 2011.

[4] K. I.-K. Wang, J. Yeh, Z. Salcic, J. Akagi, and D. Wlodkowic, 'FPGA-based Bio-cybernetic Automation System for Lab-on-a-chip Zebrafish Embryo Arrays', Int J Intell Syst Technol Appl, vol. 13, no. 1/2, pp. 17-36, Feb. 2014.

[5] J. Akagi, F. Zhu, C. J. Hall, K. E. Crosier, P. S. Crosier, and D. Wlodkowic, 'Integrated chip-based physiometer for automated fish embryo toxicity biotests in pharmaceutical screening and ecotoxicology', Cytom. Part J. Int. Soc. Anal. Cytol., vol. 85, no. 6, pp. 537547, Jun. 2014.

[6] K. M. Henry, L. Pase, C. F. Ramos-Lopez, G. J. Lieschke, S. A. Renshaw, and C. C. ReyesAldasoro, 'PhagoSight: an open-source MATLAB ${ }^{\circledR}$ package for the analysis of fluorescent neutrophil and macrophage migration in a zebrafish model', PloS One, vol. 8, no. 8, p. e72636, 2013.

[7] C. C. Reyes-Aldasoro, 'Bright Field Microscopy', in Biomedical Image Analysis Recipes in $M A T L A B \circledR$, John Wiley \& Sons, Ltd, 2015, pp. 215-289.

[8] J. Serra, 'Introduction to mathematical morphology', Comput. Vis. Graph. Image Process., vol. 35, no. 3, pp. 283-305, Sep. 1986.

[9] O. H. Schmitt, 'A thermionic trigger', J. Sci. Instrum., vol. 15, no. 24, pp. 24-26, 1938.

[10] N. Otsu, 'A Threshold Selection Method from Gray-Level Histograms', IEEE Trans. Syst. Man Cybern., vol. 9, no. 1, pp. 62-66, Jan. 1979.

[11] C. C. Reyes-Aldasoro, S. Akerman, and G. M. Tozer, 'Measuring the velocity of fluorescently labelled red blood cells with a keyhole tracking algorithm', J. Microsc., vol. 229, no. Pt 1, pp. 162-173, Jan. 2008.

[12] S. de Oliveira, C. C. Reyes-Aldasoro, S. Candel, S. A. Renshaw, V. Mulero, and A. Calado, 'Cxc18 (IL-8) mediates neutrophil recruitment and behavior in the zebrafish inflammatory response', J. Immunol., vol. 190, no. 8, pp. 4349-4359, Apr. 2013.

[13] L. Pase, J. E. Layton, C. Wittmann, F. Ellett, C. J. Nowell, C. C. Reyes-Aldasoro, S. Varma, K. L. Rogers, C. J. Hall, M. C. Keightley, P. S. Crosier, C. Grabher, J. K. Heath, S. A. Renshaw, and G. J. Lieschke, 'Neutrophil-delivered myeloperoxidase dampens the hydrogen peroxide burst after tissue wounding in zebrafish', Curr. Biol., vol. 22, no. 19, pp. 18181824, Oct. 2012.

[14] F. de Chaumont, S. Dallongeville, N. Chenouard, N. Hervé, S. Pop, T. Provoost, V. MeasYedid, P. Pankajakshan, T. Lecomte, Y. Le Montagner, T. Lagache, A. Dufour, and J.-C. Olivo-Marin, 'Icy: an open bioimage informatics platform for extended reproducible research', Nat. Methods, vol. 9, no. 7, pp. 690-696, Jul. 2012.

[15] M. Rashid, M. Frasca, A. Ali, R. Ali, L. Fortuna, and M. Xibilia, 'Artemia swarm dynamics and path tracking', Nonlinear Dyn., vol. 68, no. 4, pp. 555-563, Jun. 2012.

[16] Y. Huang, C. C. Reyes Aldasoro, G. Persoone, and D. Wlodkowic, 'Integrated microfluidic technology for sub-lethal and behavioral marine ecotoxicity biotests', in Proc. SPIE 9518, 2015.

[17] S. R. Kirchner, M. Fedoruk, T. Lohmüller, and J. Feldmann, 'Analyzing the Movement of the Nauplius “Artemia salina” by Optical Tracking of Plasmonic Nanoparticles', J. Vis. Exp., no. 89, p. e51502, Jul. 2014. 